\title{
Cooperation Model with Costly Punishment
}

\author{
T.M. Gradowski ${ }^{a}$, M.J. Mrowinski ${ }^{a}$ And R.A. Kosinski ${ }^{a, b}$ \\ ${ }^{a}$ Warsaw University of Technology, Faculty of Physics, Koszykowa 75, Warszawa 00-662, Poland \\ ${ }^{b}$ Central Institute of Labor Protection - National Research Institute, Czerniakowska 16, 00-701 Warszawa, Poland
}

In recent years the issue of costly punishment in systems where free-riding occurs has been a subject of extensive research in the field of game theory. This issue is present in many areas of human activities like paying taxes, using public transport, wireless Internet or P2P networks. It is one of the most common dilemmas in modern societies. In this work we present a simple model of cooperation with three possible strategies (cooperate, defect or punish). In the model players explore the available strategies according to their interactions with other players. We introduce two groups of parameters. The first group is sociological-like and it describes the social acceptance for the free riding behavior. The other group of parameters describes the economical aspects of the system - the cost and efficiency of punishers. Using differential equations approach, as well as an agent based model, we look for equilibrium properties of the system.

PACS: 87.23.Ge, 89.65.--s

\section{Introduction}

Human societies during the process of evolution developed very complex forms of cooperation. From collective hunting in the Stone Age to modern public health care systems, voluntary cooperation is one of the most important indicators of civilization. In general, cooperation can be described as a process in a society, the members of which are willing to participate, i.e. to pay some cost, in order to maintain some public good. The only purpose of the public good is to bring benefits to its contributors. However, as soon as any public good forms, the free riding issue arises. Free riders are members of the society who are not contributing to the public good but are still drawing benefits from it. If free-riding becomes the dominant strategy, the public good vanishes. Thus preventing free riding is a big challenge for societies. Free riding problem has been recognized in many areas of our life: avoidance of tax payment or health care insurance premiums, using public transport without paying for tickets, exploiting natural resources, poaching or wireless network leeching. In order to prevent this issue, various forms of punishing are introduced. Basically, punishing means paying additional cost by contributors to punish the wrong-doers by reducing their payoff. Thus punishment is an altruistic act which helps to keep the public good.

One of the most popular models describing the cooperation problem is Public Good Game (see [1] for a review). In this game a large population creates a public good which is distributed equally among all members of the group. The contributions that create the public good are paid by the players voluntary. It has been proven experimentally $[2,3]$ that in systems without any form of coercion or punishment the players tend not to participate, the public good reduces and finally vanishes. The experiments have shown that altruistic punishment plays an important role in such systems.
In recent years the issue of costly punishment has been a subject of research in the field of game theory [4-6] . Game-theoretic models assume that strategies spread according to individual rational motivations. Players adopt new strategies in order to maximize the utility function (e.g. payoff).

However, there is another approach in which opinions, cultures, languages can spread in a population as a direct result of individual personal interactions (in a similar way to epidemics) according to some transition probabilities. For example, individuals can change their opinion under the influence of social impact like in majority rule model or voter model (for review see [7]) or can get infected with a disease from one of their neighbors [8].

In this paper we investigate the equilibrium issue in the systems with punishment using the epidemiological approach. We propose both differential and agent-based models and compare the outcome.

\section{The model}

In order to characterize the process of spreading of strategies in a public good game we propose a mean field differential system describing the transitions between available strategies among the players. The allowed strategies are: contribute $(C)$, free ride $(F)$ and punish $(P)$.

We assume 4 types of transitions of strategies according to the interactions with other agents. These transitions are:

- $\alpha$ (Free rider $\rightarrow$ Contributor) - free riders change their strategy to contribute after interaction with punishers with probability $\alpha$ ( $\alpha$ is the punishing efficiency parameter).

- $\beta$ (Punisher $\rightarrow$ Contributor) - punishers become contributors with probability $\beta$, which is the cost parameter of punishing. 
- $\gamma$ (Contributor $\rightarrow$ Free rider) - contributors are tempted to free ride; they change their strategy to free riding with probability $\gamma$ after interactions with other free riders.

- $\delta$ (Contributor $\rightarrow$ Punisher) - contributors become irritated by the destructive behavior of free riders and change their strategy to punish with probability $\delta$ after meeting free riders.

Parameters $\alpha, \beta, \gamma$ and $\delta$ have a meaning of transition probabilities. Parameters $\alpha$ and $\beta$ refer to economical properties of the system (cost and efficiency). Parameters $\gamma$ and $\delta$ apply to sociological conditions of the population (social acceptance for harmful behavior). The main equations take the following form:

$$
\left\{\begin{array}{l}
\frac{d C}{d t}=P F \alpha+P(1-F) \beta-C F(\delta+\gamma) \\
\frac{d F}{d t}=C F \gamma-P F \alpha \\
\frac{d P}{d t}=C F \delta-P(1-F) \beta
\end{array}\right.
$$

These equations obey the Lotka-Volterra preypredator model assumptions. The most obvious difference is the presence of the second term of the third equation, which corresponds to the decrease of the number of punishers caused by the lack of contributors. This decrease is connected with the cost of the punishing process ( $\beta$ parameter): not necessary and expensive punishers are removed from the population (they are converted to fair contributors).

This model has two trivial unstable fixed points: $(C, F, P)=(1,0,0)$ and $(C, F, P)=(0,1,0)$. The third fixed point is stable:

$$
\left\{\begin{array}{l}
C^{*}=\frac{\delta \alpha^{2}}{\delta \alpha^{2}+\beta \gamma^{2}+\alpha \gamma(\beta+\delta)} \\
F^{*}=1-C^{*}\left(\frac{\gamma}{\alpha}+1\right) \\
P^{*}=\frac{\gamma}{\alpha} C^{*}
\end{array}\right.
$$

An alternative approach to the above problem is an agent based model. We put all agents on a square lattice with periodic boundary conditions. Each agent is able to interact with his or her four neighbors. In order to explore well-mixed system the agents perform random walk over the lattice. High mobility makes the spatial distribution irrelevant for the dynamics, it prevents the formation of spatial patterns and makes it possible to compare agent based model with the mean field approach.

At the beginning of the simulation one of the available strategies is randomly chosen for each agent. The simulation process goes as follows:

- agents are selected according to the shuffle-update rule
- each agent selects one neighbor at random and changes his or her strategy according to the rules presented at the beginning of this section

- at the end of each time step agents are shuffled one more time and each agent changes his or her position on the lattice with one randomly selected neighbor.

\section{Results}

In this section we present the results of both models for a number of sets of parameters. To visualize the outcomes we plot 3-dimensional phase diagrams on ternary graphs (Fig. 1). On the left column results of the mean field model are shown. Arrows represent the directions of the $[d C, d F, d P]$ vectors, the values of which were obtained using the Euler's method. Colors represent normalized vector lengths (black is the lowest). The asterisk symbol represents the stable fixed point from Eq. 2. On the right column results from the numerical agent based model are depicted. Each plot comprises a set of trajectories for different initial conditions. Asterisk in the right column corresponds the stable point from the differential model for the sake of comparison.

As an example, we present three representative cases of cooperation scenarios corresponding to three different sets of parameters:

- low cost, high free riding acceptance - if the cost parameter $\beta$ and the irritation parameter $\delta$ were zero, the system would have an infinite number of stable fixed points located on the straight line $P=C$ (equal number of contributors and punishers). For $\beta$ and $\delta$ close to zero, the system tends to the situation where the number of punishers and contributors is equal and then slowly moves towards the fixed point.

- in case of high temptation, low cost and efficiency the system is dominated by free riders.

- when free riding acceptance and temptation are low, cost and efficiency are high (the economical and sociological condition of the population is good), the system is dominated by contributors.

Although our model is similar to cyclic Lotka-Volterra model with three species or rock-scissors-paper game (for review see [9]), it exhibits different dynamic scenarios than it's prototypes. The dynamics of the model (with non-zero parameters) always leads to the stable fixed point, where all strategies are present in the population. This fixed point corresponds to the Nash equilibrium of the system, speaking in the game-theoretical language. Such a behavior is similar to the Traulsen et al. model [6] in the case of high mutation rate, that is high probability of random exploration of strategies. However our model also contains unstable fixed points - saddle points, 

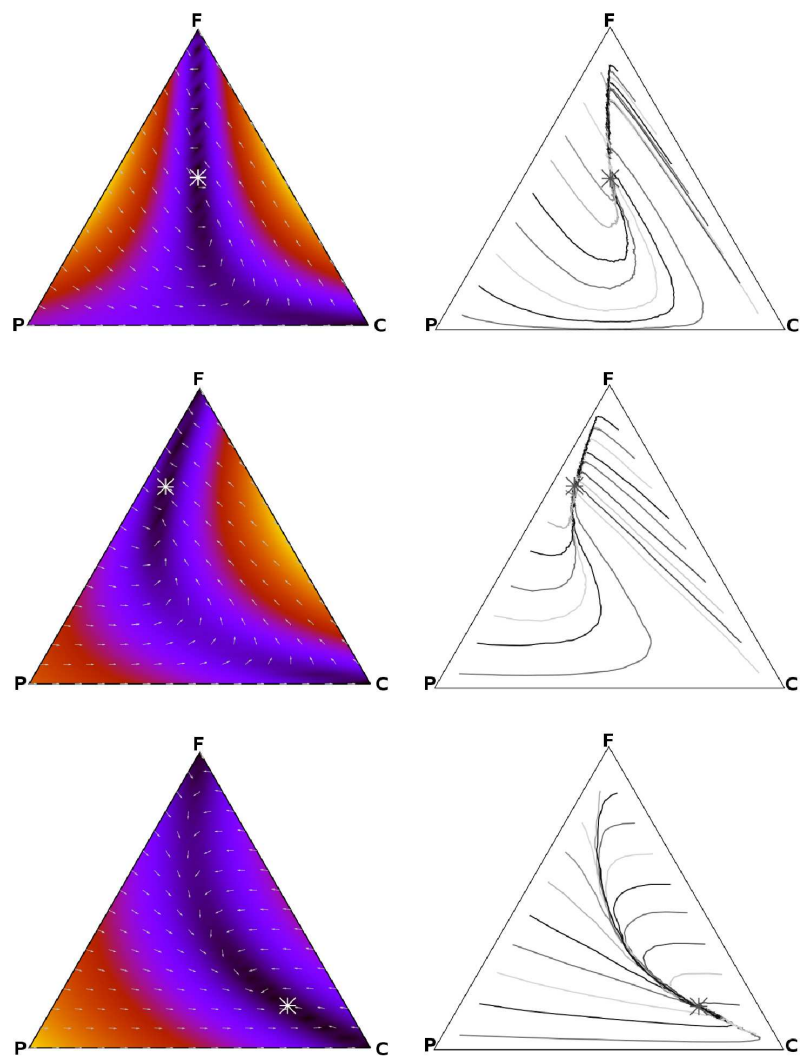

Fig. 1. Results from the mean field differential model (left column) and agent based model with random walk (right column) for the following sets of parameters (top to bottom): $\alpha=0.1, \beta=0.01, \gamma=0.1, \delta=0.01$ (low cost, high freeriding acceptance); $\alpha=0.05, \beta=0.05$, $\gamma=0.2, \delta=0.1$ (high temptation, low cost and efficiency); $\alpha=0.2, \beta=0.2, \gamma=0.05, \delta=0.3$ (low freeriding acceptance, low temptation, high cost and efficiency). Light color corresponds to fast dynamics, dark color to slow dynamics.

which are attractive in one direction and repulsive in the other. These points are $C=1$ and $F=1$.

Our model doesn't reveal any orbits, which are present in other previously mentioned models $[4,5]$. In these models fourth stategy is introduced - non-participants. These players, also called loners, are not taking part in the enterprise, they don't participate but also they don't draw any benefits from the public good. In such systems periodic orbits are present in the absence of the punishers. This behavior is not possible in our model of three strategies.

\section{Conclusions}

Models presented in previous sections, despite their simplicity, provide reasonable results that correspond with common sense and intuition. Agent based model of random walkers shows a fine agreement with the mean field approach based on differential equations.

This paper is an attempt to propose an alternative approach to the problem of spreading of cooperative strategies in populations. Game-theoretic methods are well established and seem to be proper for cooperation phenomena. In our opinion, approach based on social impact theory, like the one presented in this paper, is complementary to models based on rational payoff-aware dynamics.

\section{Acknowledgements}

This work was supported by the European Cooperation in the field of Scientific and Technical Research COST Action MP0801 "Physics of competition and conflicts".

\section{References}

[1] K. Sigmund, Trends in Ecology and Evolution 22, 11 (2007).

[2] E. Fehr, S. Gachter, Nature 415, 137 (2002).

[3] E. Fehr, S. Gachter, Am. Econ. Rev. 90, 980 (2000).

[4] J. H. Fowler, Proc. Natl. Acad. Sci. USA 102, 19 (2005).

[5] H. Brandt, C. Hauert, K. Sigmund, Proc. Natl. Acad. Sci. USA 113, 2 (2006).

[6] A. Traulsen, C. Hauert, H. De Silva, M. A. Nowak, K. Sigmund, Proc. Natl. Acad. Sci. USA 106, 3 (2009).

[7] C. Castellano, S. Fortunato, V. Loreto, Rev. Mod. Phys. 81, 591 (2009).

[8] R. Pastor-Satorras, A. Vespignani, Phys. Rev. E 63, 066117 (2001).

[9] E. Frey, Physica A 389, 4265 (2010). 